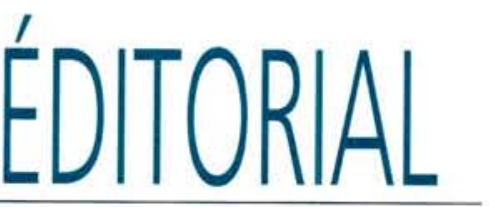

MARCEL JOLLIVET, JEAN-MARIE LEGAY, GÉRARD MÉGIE

\title{
Pollution médiatique
}

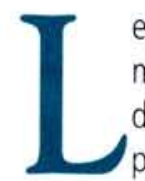

es résultats d'une enquête épidémiologique, conduite sous l'égide du Réseau national de santé publique (RNSP) dans le cadre du programme de recherche européen APHEA (Air Pollution and Health) et traitant des incidences sur la santé humaine de la pollution de l'air en milieu urbain, viennent de faire la "Une" de tous les médias. Un grand journal d'information qui se targue de sérieux titrait ainsi : "Plusieurs centaines de décès sont imputables à la pollution de l'air" (Le Monde, 6 février 1996). Reprise de la même manière par l'ensemble de la presse, cette annonce a pu laisser croire que des résultats entièrement nouveaux venaient aujourd'hui démontrer de façon quantitative les risques provoqués par la pollution atmosphérique. Or il n'en est rien. Les auteurs de ce rapport n'ont pas mis en évidence de relation directe de cause à effet entre pollution de l'air et mortalité au sein de l'ensemble de la population. Ils ont simplement montré que la pollution atmosphérique pouvait être considérée comme un facteur aggravant, susceptible d'entraîner une surmortalité chez des personnes souffrant déjà d'insuffisances respiratoires chroniques, dont la probabilité de décès est élevée et qui peuvent mourir à tout moment du fait d 'événements mineurs de la vie courante. Les résultats statistiques montrent qu'il est "possible" que, dans ces conditions, la pollution atmosphérique joue un rôle. Ce résultat n'est d'ailleurs pas nouveau. L'étude ERPURS effectué par I'Observatoire Régional de la Santé d'lle de France en 1994, et sur laquelle d'ailleurs l'étude du RNSP se fonde, avait déjà mis en évidence ce rôle aggravant de la pollution atmosphérique. La seule nouveauté de l'étude du RNSP tient à la méthode statistique utilisée. II ne nous appartient pas d'en discuter la validité, mais il importe de constater que ces résultats sont publiés dans la grande presse avant même de l'être dans la littérature scientifique, dont les critères de publication sont fondés sur l'évaluation par les pairs. On peut aussi remarquer qu'aucune des treize autres villes européennes associées au projet n'a opté pour la même méthode.

Comment alors expliquer le passage de la précision et de la rigueur d'un résultat scientifique à une formulation qui est de fait de la désinformation?

De l'aveu même des auteurs de l'enquête, I'appel aux médias est délibéré, puisqu'il s 'agit de "créer un lobby de la santé publique pour contrer celui de l'automobile, au moment crucial où notre pays engage le débat sur les moyens de réduire la pollution » (Libération, 8 février 1996). Les épidémiologistes auteurs de l'étude ont donc opté pour " un effet de sidération à grand coup de morts statistiques " (ibid.). La fin justifie ainsi les moyens, et l'éthique scientifique est quelque peu mise à mal ! Personne n'est donc innocent dans cette affaire, ni les scientifiques concernés, ni les médias.

Plutôt que de jouer sur un réflexe de peur des citoyens, mieux vaudrait souligner la nécessité d'affiner la connaissance de la composition de l'air urbain. Parmi les polluants qui y sont présents, l'enquête en cause ne prend en compte que le dioxyde de soufre $\mathrm{SO}_{2}$ et les poussières, qui provoquent des effets toxiques à court terme. Or il importe de remarquer que les émissions de $\mathrm{SO}_{2}$ dans l'atmosphère ont très fortement diminué au cours de la dernière décennie. Par contre, les oxydes d'azote et l'ozone n'y sont pas considérés, alors même que leurs concentrations augmentent fortement dans les atmosphères urbaines lors des pointes de pollution, et dépassent largement celle du dioxyde de soufre.

Enjeux économiques, enjeux politiques, enjeux de santé publique, phénomènes complexes à analyser, recours légitimant à la science, tous les ingrédients constitutifs d'un problème d'environnement sont bel et bien là. Ce problème de la pollution de l'air urbain apparaît ainsi comme un cas d'école de ces interférences qu'il faut mettre en évidence pour éviter les malentendus et le trop facile et dangereux recours au catastrophisme et à la dramatisation. C'est un sujet sur lequel il est nécessaire que Natures, Sciences, Sociétés revienne. 